\title{
Experimental observation of visible flame propagation rate in accidental deflagration explosions and explosive load reduction.
}

\author{
Alexander Komarov* and Nikolay Gromov \\ Moscow State University of Civil Engineering, Yaroslavskoe shosse, 26, Moscow, 129337, Russia
}

\begin{abstract}
The article reveals the results of experimental studies of the impact exerted by an accidental explosion scenario on the visible flame propagation rate. The numerical value of the visible flame propagation rate defines the parameters of the explosive load accompanying an accidental explosion. The conducted experimental studies were used for justification of recommendations aimed at reduction of potential explosive loads during accidental events.
\end{abstract}

\section{Introduction}

The main parameters defining explosive pressure during deflagration explosions include visible flame propagation rate and the fireball radius (of explosion products). During explosive combustion there emerge gas-dynamic flows which cause the mixture to burn faster, i.e. increase the visible flame propagation rate and speed up diffusive processes inside the mixture. This leans the mixture at the fireball's boundary which in its turn results in a lower flame velocity and decreased fireball dimensions. The maximum effect of the above mentioned processes on explosive load formation can be observed at the boundary of the explosive cloud [1].

To prove this, there was conducted a series of experimental studies aimed at measuring the flame propagation rate which allowed to calculate the acceleration process of explosive combustion, as well as its deceleration at the end of the explosion [2].

\section{Methods}

Stoichiometric propane-air mixture (with the fuel concentration of $4.5 \%$ vol.) was used to conduct the experiments. The experimental explosions were performed in a stretched out $4.12 \mathrm{~m}$ chamber with a square $0.16^{*} 0.16 \mathrm{~m}$ section. The plan view of the chamber is shown in Figure 1.

The chamber was divided into three parts with the help of extending plates. The ends of the chamber remained open during the experiment. A stoichiometric mixture was generated in the central chamber with the help of a measuring syringe. The mixture was ignited by a continuously sparking source in various places $\left(T_{1}\right.$ in the center of the chamber and $T_{2}$ and $\mathrm{T}_{3}$ at the sides) demonstrated in Figure 1. Hereinafter, we will refer to the experiment with 
mixture ignition in point $T_{1}$ as Experiment $I$, with ignition in point $T_{2}-$ as Experiment II and with ignition in point $\mathrm{T}_{3}$ - as Experiment III.

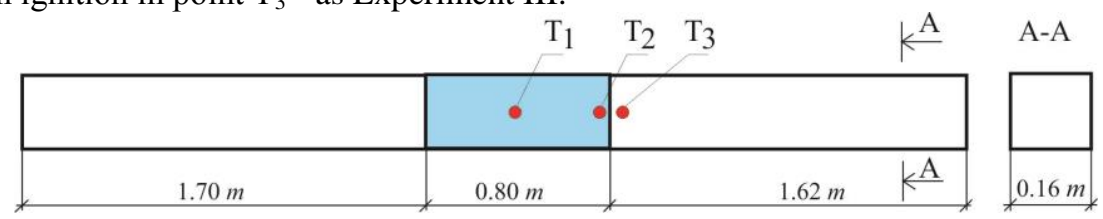

Fig. 1. The plan view of the experimental chamber.

The process of explosive combustion was filmed by a slow-motion camera (290 frames per second), based on the acquired videos there was defined the flame front location versus time characteristic. Then, there were determined all kinematic parameters of the flame front in axial direction: the flame velocity versus time and flame velocity versus coordinate characteristics.

Figure 2 provides several moments of the flame propagation process registered during Experiment I.
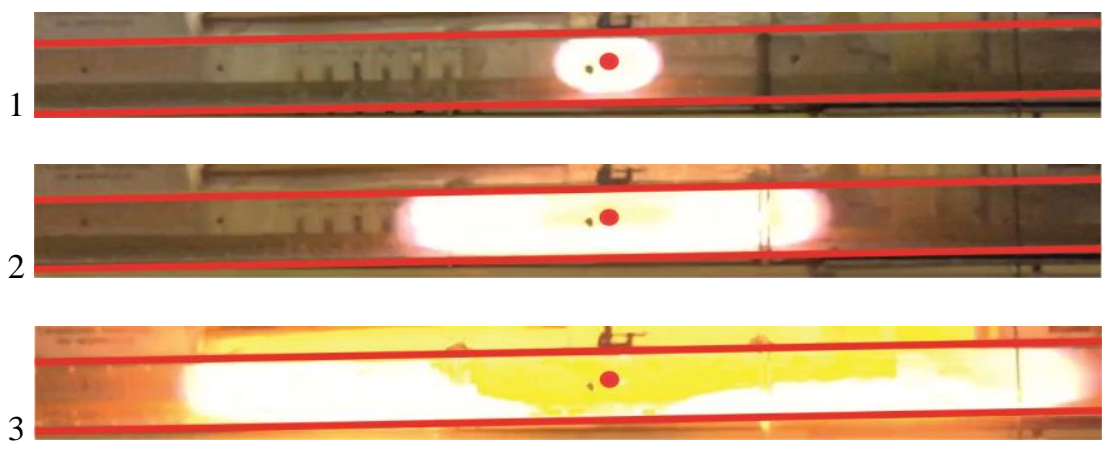

Fig. 2. Photos of the flame propagation process.

Experiment I. Mixture ignition in the center of an explosive cloud (point $\mathrm{T}_{1}$ ). 1 - in $29.3 \mathrm{~ms} ; 2$ - in $54.3 \mathrm{~ms} ; 3$ - in $75.2 \mathrm{~ms}$ after mixture ignition.

Experimental coordinates of the flame front location for various instants of time which were acquired from photos of the explosion process (see Figure 2) are given in Figure 3. The numeric value of $\mathrm{X}$ corresponds to the distances from the ignition place - $\mathrm{T}_{1}$ to the left visible flame front along the camera's axis. The acquired relations should be differentiated in order to determine the velocity characteristics of the flame front. Direct differentiation of experimental points entail a significant margin of error. That is why the acquired experimental values of flame front location in time were interpolated first.

The accuracy of the $\mathrm{X}$ coordinate measurement depends much on the subjective evaluation of the flame front boundary by a person processing the experiment. However, with regard to this problem, this is the only way to experimentally determine the dynamic parameters of the flame front during an explosion. A portion of criticisms may also be addressed to the one-dimensional model used in the experiment. But the simulation tests of deflagration explosions in the open space that have been previously conducted in Russia and abroad have not given practically essential answers to multiple questions related to flame front dynamics during accidental explosions [3]. 

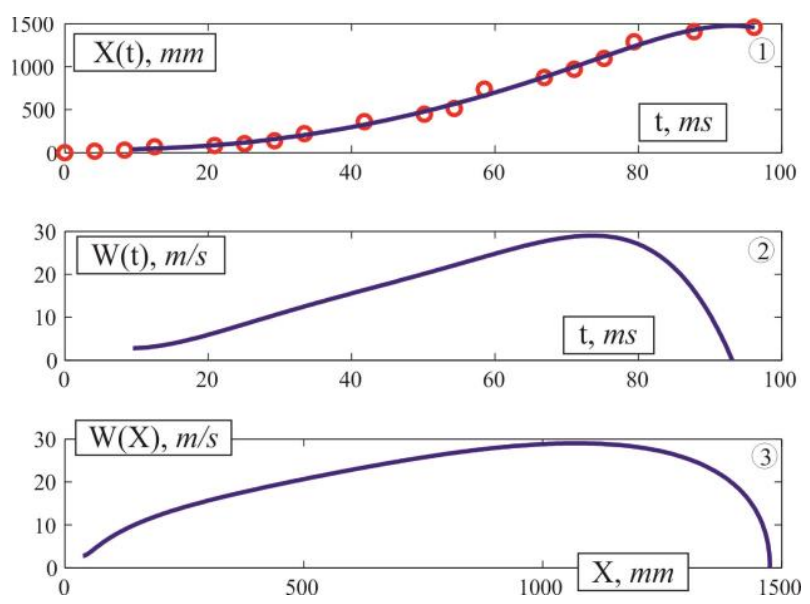

Fig. 3. Dynamic parameters of the flame front. Experiment I.

1 - flame from location for various instants in time and interpolatory flame front location versus time characteristic; 2 - flame velocity versus time characteristic; 3 - flame velocity versus coordinate characteristic.

Dynamic parameters of the flame front (see Figure 3) suggest that at the initial stage of the explosion it accelerates but then slows down until it stops completely (the end of combustion process).

Based on the results of Experiment I, the following conclusions may be made. Considering there was conducted an explosion of stoichiometric propane-air mixture, we may assume that during complete combustion its initial volume shall increase 8.0 times [1]. Thus, the linear dimensions of the explosion product cloud should constitute $0.8 \cdot 8.0=6.4 \mathrm{~m}$ while the maximum value of $\mathrm{X}$ coordinate should be $3.2 \mathrm{~m}$. The experiments demonstrated that the maximum value of the visible flame propagation rate is registered at $\mathrm{X}=1.0 \ldots 1.2 \mathrm{~m}$ (see Figure 3), which is three times less. At this point, the maximum flame velocity reached $25-30 \mathrm{~m} / \mathrm{s}$.

Let's consider results of Experiment II with mixture ignition performed at the right side of gas-vapor cloud (point $T_{2}$, see Figure 1). Figure 4 provides experimental coordinates of the flame front locations for various instants in time, which were acquired from the photo sequences of the explosion process, as well as velocity characteristics of the flame front acquired from interpolation ratios describing the flame front location versus time characteristic.

The given data suggests that if the mixture gets ignited at the side of the cloud, the visible flame propagation rate is at least two times lower than when the ignition occurs in the center. In the previous experiment, the maximum flame velocity reached $25-30 \mathrm{~m} / \mathrm{s}$ while in this experiment it did not exceed $12-14 \mathrm{~m} / \mathrm{s}$. Thus, we can state that if the mixture gets ignited at the side of the cloud, the visible flame propagation rate is at least two times lower than when the ignition occurs in the center of the cloud. 


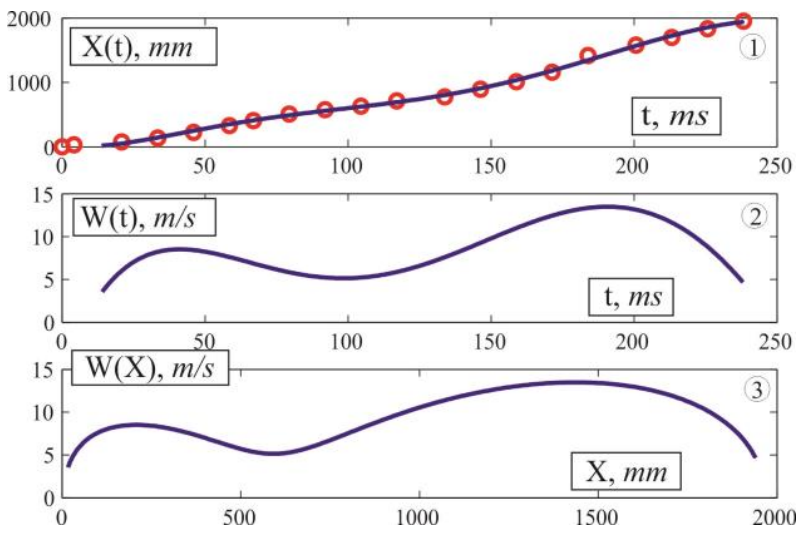

Fig. 4. Dynamic parameters of the flame front. Experiment II.

1 - experimental locations of the flame front and interpolatory flame front versus time characteristic; 2 - flame velocity versus time characteristic; 3 - flame velocity versus coordinate characteristic.

Let's consider results of Experiment III with mixture ignition source located outside the explosive cloud (point $T_{3}$, see Figure 1). In this experiment, the mixture ignition was performed in some time after plates dividing the chamber into three sections were removed. The ignition source located at $T_{3}$ was continuously giving up sparks while the mixture was drifting towards it due to diffusion after the removal of the separation plates. At a certain moment, when the explosive mixture reached $\mathrm{T}_{3}$, there occurred an explosion. To a certain extent, this very experiment emulates the drifting process of the explosive cloud and its ignition by a constantly active source. With regard to emergency situations, it is a quite typical scenario for an explosive accident. The main difference of initial conditions of Experiment III from the conditions of Experiment II is that the latter involves an explosion of a stoichiometric mixture, while the former implies initial ignition of a lean mixture created during diffusive drifting of the cloud towards ignition source $\mathrm{T}_{3}$.

Figure 5 provides experimental coordinates of the flame front locations for various instants in time, as well as velocity characteristics of the flame front acquired from the interpolation ratio describing the flame front location versus time characteristic in Experiment III.

The given data suggests that the maximum flame velocity within Experiment III constituted no more than $5 \mathrm{~m} / \mathrm{s}$. Thus, we can state that in case of a drifting explosive cloud or when it spreads out due to diffusion, the mixture ignition at the side of the cloud by a continuously operating source leads to a deflagration explosion with a visible flame propagation rate of $5-10 \mathrm{~m} / \mathrm{s}$. The visible propagation rate remains almost unchanged as the explosion develops. In some cases, there may be a firestorm which is not accompanied by excessive pressure and is marked only by short-term heat loads. A person affected by the firestorm mostly suffers burns of lungs and upper respiratory airways. Objects affected by the firestorm do not catch fire as the heat impact is short-lived.

Based on the conducted experiments, there may be provided the following recommendations regarding explosive load reduction. 

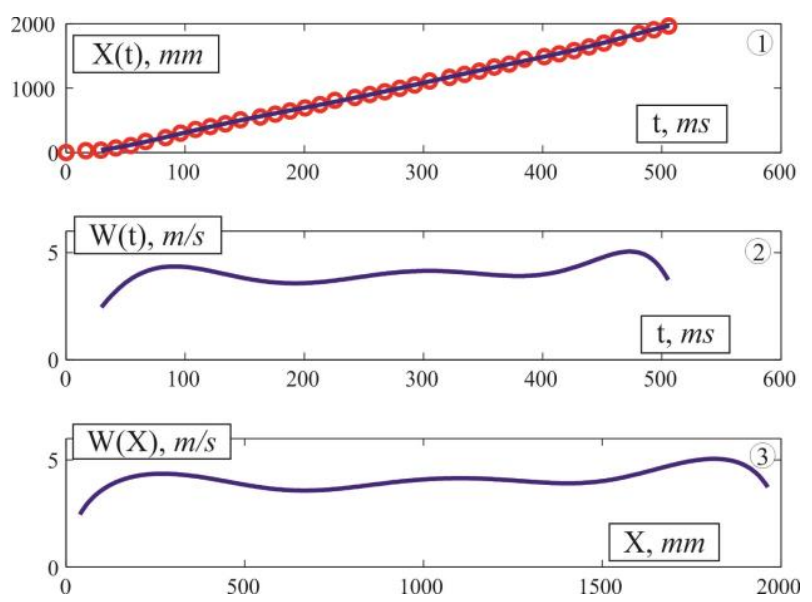

Fig. 5. Dynamic parameters of the flame front. Experiment III.

1 - experimental locations of the flame front and interpolatory flame front versus time characteristic; 2 - flame velocity versus time characteristic; 3 - flame velocity versus coordinate characteristic.

The analysis of experimental studies demonstrated that the visible flame velocity decreases at least two times if the cloud gets ignited at its boundary compared to the velocity of explosive combustion when the ignition starts from the center. This very peculiarity of deflagration explosion development allows to formulate the following measure aimed at reducing the explosive load generated by a propagating ground drifting explosive cloud. Ground drifting explosive clouds emerge from spills of highlyinflammable liquids (HIL), when there occur significant leaks of heavy hydrocarbon compounds, etc. If the atmosphere is not completely stable, the cloud may drift towards facilities which are subject for protection. An arbitrary ignition source might appear randomly, including the least favorable locations, for example, in the center of the explosive cloud, which will lead to significant explosive loads. If there is "a controllable ignition spot", for example, in a form of continuously sparking source located between the emergency facility and the protected territory, the ignition of the ground drifting cloud will start from its side; this will not entail acceleration of combustion, nor will it involve significant flame velocities or high explosive loads.

Let's provide the following calculation results to justify the recommended explosive load reduction measures. For example, let's consider a scenario when there occurs an explosion of diesel fuel vapors stored in a standard $40 \mathrm{~m}^{3}$ tank. The emergency situation is caused by probable depressurization of the tank and a fuel spill which generates a drifting explosive cloud as it gradually evaporates. In this case there might occur a deflagration explosion with the maximum fireball's radius of no more than $10 \mathrm{~m}$ and the maximum flame velocity of no more than $W=107.2 \mathrm{~m} / \mathrm{s}$. The indicated parameters of the deflagration explosion are registered in case the ignition occurs in the least favorable location [4]. If positive ignition by a continuously operating source located between the accident site and the protected facility is provided, the drifting cloud gets ignited at the moment when its side reaches the sparking source. In this case the flame velocity will be two times lower and its actual value will not exceed $W=53.6 \mathrm{~m} / \mathrm{s}$.

There was considered a pattern of relatively even distribution of fuel concentration in a drifting cloud. Kinematic characteristics of the flame front for the relatively even concentration distribution inside the cloud are shown in Figure 6.

At the initial stage of the explosion the flame velocity is minimal and equals $W_{M I N}$, i.e. It is assumed there is no flame intensification or acceleration at this stage. Thus, if $0<R<A_{1}$ $\cdot R_{F B}$ ( $R$ is current fireball's radius characteristic, $R_{F B}$ is the maximum fireball radius value), 
it was assumed that the visible flame propagation rate was $W_{M I N}$. In case $A_{1} \cdot R_{F B}<R<\left(A_{1}+A_{2}\right) \cdot R_{F B}$, the visible flame propagation rate rises evenly to $W_{M A X}$, i.e. the flame accelerates (the cause of the acceleration in this case is not important) while for $\left(A_{1}+A_{2}\right) \cdot R_{F B}=\left(1-A_{3}\right) \cdot R_{F B}<R<R_{F B}$ the visible flame propagation rate decreases from $W_{M A X}$ to 0 .

While $A_{1}$ is the initial portion of lifetime of fireball $A$, with a constant visible flame velocity, $A_{2}$ is the interim phase of the fireball's lifetime with an increasing visible flame velocity, $A_{3}$ is the fireball's decay stage with a decreasing visible flame velocity $\left(A_{1}+A_{2}+A_{3}=1\right)$. The following parameter values were assumed in the calculations: $A_{1}=0,10$; $A_{2}=0,8 ; A_{3}=0,10$.
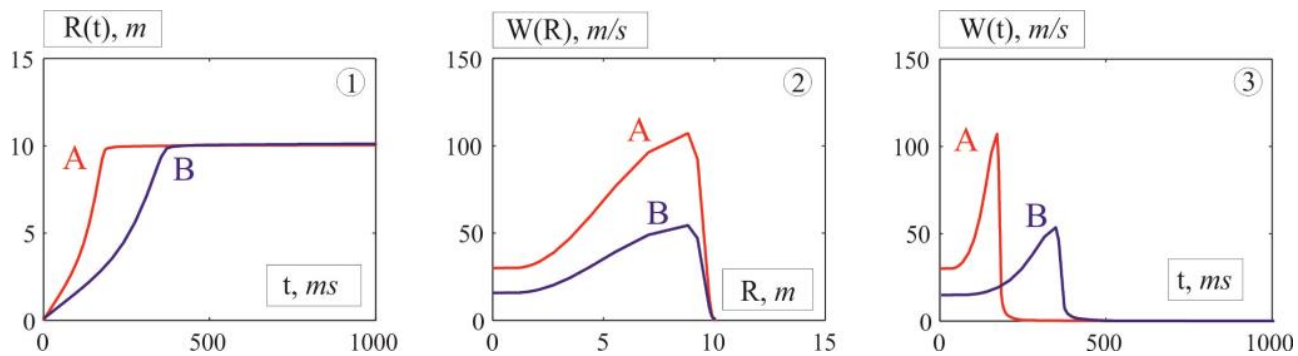

Fig. 6. Dynamic parameters of the fireball.

1 - flame front coordinate versus time characteristic; 2 - visible flame propagation rate versus flame front position characteristic; 3 - visible flame propagation rate versus time characteristic. A - arbitrary ignition source; B - controllable ignition spot.

The accident scenario involving an arbitrary ignition source located in the least favorable area of the drifting cloud leads to a deflagration explosion with the maximum fireball radius of $R_{F B}=10 \mathrm{~m}$ and the maximum flame velocity of $W=107.2 \mathrm{~m} / \mathrm{s}$, (curve A in Figure 6). The accident scenario involving an ignition from a continuously operating source located at the edge of the cloud leads to a deflagration explosion with the fireball radius of under $R_{F B}=10 \mathrm{~m}$ and the maximum flame velocity of $W=53.6 \mathrm{~m} / \mathrm{s}$, (curve B in Figure 6). Figure 7 demonstrates arbitrary ignition of mixture ' $A$ ' as well as positive ignition of mixture ' $\mathrm{B}$ ' performed by special equipment generating a continuously sparking protection belt around the protected facility.
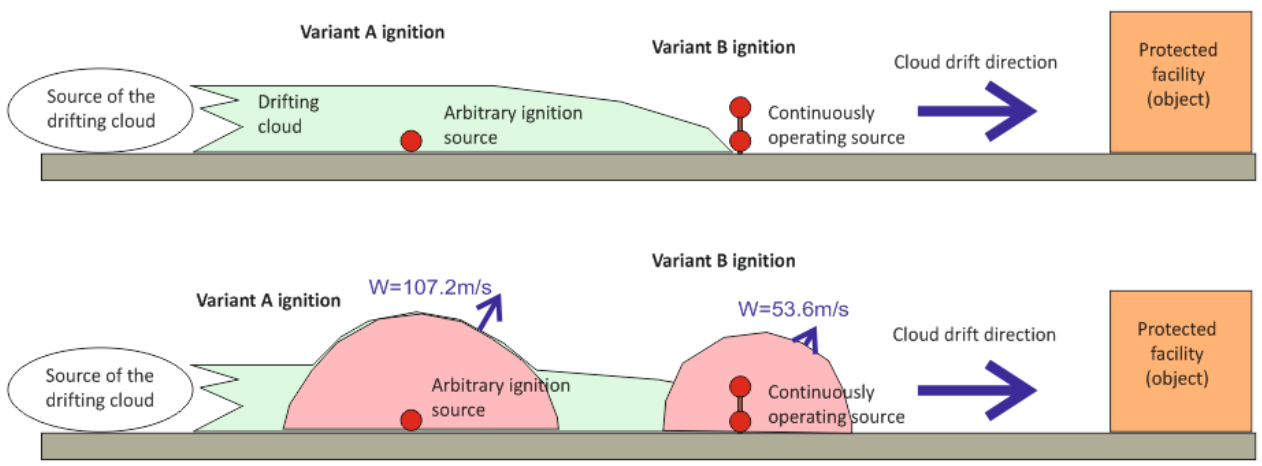

Fig. 7. Illustrations of a recommended method of explosive combustion velocity reduction in a drifting cloud.

«1» - arbitrary ignition ' $A$ ' and recommended ignition device ' $B$ ' patterns.

«2»- explosion area in case of arbitrary ' $A$ ' and positive 'B' ignition of a drifting cloud.

Explosive pressure time laws at the distances of $10 \mathrm{~m}$ (fireball's boundary) and $20 \mathrm{~m}$ are given in Figure 8. 


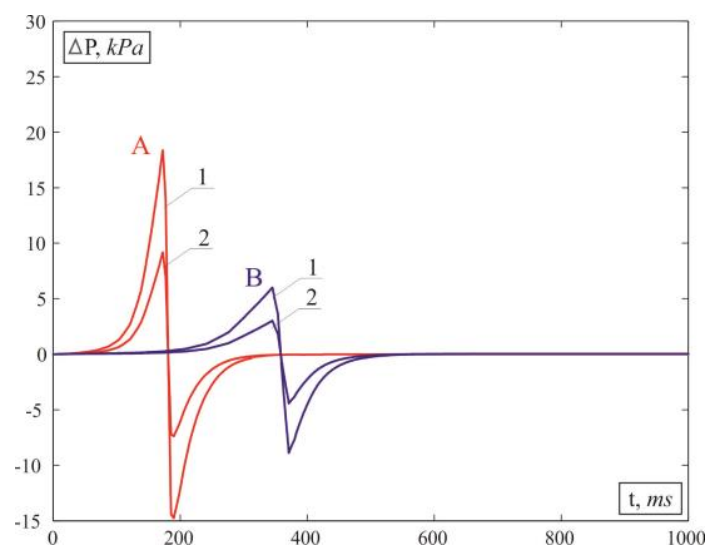

Fig. 8. Calculated excessive pressure versus time characteristic at the distances of: $1-10 \mathrm{~m} ; 2-20 \mathrm{~m}$ from the explosion site.

A - explosion in case of arbitrary ignition of a drifting cloud; B - positive ignition of a ground drifting cloud.

The results of the conducted calculations suggest that if the drifting cloud is ignited by a continuously operating source located between the accident site and the protected facility, i.e. at the side of the cloud drifting towards the protected facility, the explosive pressure drops at least two times.

The given calculations, as well as results of experimental studies and the analysis of real accidents that involved a drifting explosive cloud support the idea that the suggested explosive load reduction method is quite efficient.

\section{Conclusions}

The experimental studies demonstrated that if the ignition source is located at the side of the explosive mixture, the visible flame velocity is two times lower compared to when the ignition source is centered. In case of a drifting explosive cloud or when it spreads out due to diffusion, and in case of mixture ignition by continuously operating sources (or fire areas), there occurs a deflagration explosion with a visible flame propagation rate, which does not accelerate, as the explosion develops, and comprises $5-10 \mathrm{~m} / \mathrm{s}$.

This very peculiarity of deflagration explosion development allows to formulate the following measure aimed at reducing the intensity of dynamic load generated by a ground drifting explosive cloud. Constantly sparking ignition sources should be placed between the potentially hazardous source and the protected area which, in case of an accident and drifting cloud development, will provide a "controllable ignition spot" for the mixture. In this case, the accidental explosion will not entail accelerated combustion and, thus, significant flame velocities and high explosive loads as mixture ignition will take place at the edge of the cloud.

\section{References}

1. Komarov A.A. Forecasting Loads and Estimating Consequences of Their Impact on Buildings and Structures. Doctoral dissertation in 05.26.03 - Fire safety and HSE (construction building). M. MSCU. (2001) 
2. Report on 'Hazard Analysis for On-Site Accidental Explosions Including an Aircraft Crash Event' Stage I. 'Validation and ratification of evaluation methods for on-site accidental explosions. Calculations of on-site accidental explosions'. M.: MSCU (2018)

3. Komarov A.A. Calculation of Gas-Dynamic Characteristics of Flows at Deflagration Explosions at Outdoor Installations. The Fire and Explosion Safety Magazine, (2002) Vol.11. No.5. p.15-18.

4. Komarov A.A., Korolchenko D.A., Gromov N.V. Hydrodynamic Calculations for Technical Review of Accidental Explosion // Fire and Explosion Safety. - (2017) T.26, No. 10. - P.36-42. DOI:10.18322/PVB.2017.26.10.36-42.

5. Khusnutdinov D.Z. et al. Accidental Explosions of Gas-Air Mixtures in Atmosphere. M.: NRU MSCU, 2014. - 80p.

6. Marshall V. Major Chemical Hazards. Mir, M., 1989.

7. Numerical Calculation of Multidimensional Problems of Gas Dynamics Edited by Godunova S.K. M. Nauka. 1976. C.400.

8. Mishuev A.V., Khusnutdinov D.Z. Modeling of Dynamic Loads Effecting Building Structures at Emergency Deflagration Explosion. MSCU, M., 2004. 